DOI:

10.1038/nrg1908

URLs

\section{RNA WORLD}

\section{MIWI}

\section{and MILI}

find small

\section{partners}

Two independent reports reveal the existence of a new class of small RNAs, which are germline-specific and interact with mammalian Piwi proteins.

To perform their diverse functions, Argonaute proteins need to team up with small RNAs, which serve as sequence-specific guides. But although small RNA partners for the Argonaute subfamily are known, partners for the germline-specific Piwi subfamily had remained elusive, as have the details of Piwi func- tion. Thomas Tuschl and Mihaela Zavolan and colleagues now show that MILI, a mouse member of the Piwi subfamily, binds 30-nt-long, single-stranded, small RNAs. Greg Hannon and colleagues show that the same types of small RNA associate with MIWI, another mouse Piwi protein. The newly identified RNA species have been called piRNAs, for 'Piwi-interacting', and are expressed exclusively in the testes, at specific stages during germ-cell development. Both groups show that piRNAs map to clusters in the mouse genome. The clusters, which do not correlate with the gene density or repeat density, vary in length and show strong strand bias - most piRNAs lie just on one strand. The most striking feature of piRNA sequence is their $5^{\prime}$ uridine bias, which is also seen in other small RNA species such as miRNAs. Although the clustering suggests that piRNAs might be processed from longer precursors, the authors found no evidence for any double-stranded structure, including miRNA-like stem loops.

The authors found piRNAs also in the human, rat and bull. Although the primary sequence does not seem to be well conserved most mouse clusters exist at syntenic locations in the human and rat.

Germline-specific expression of Piwi proteins and piRNAs suggests that they have a role in gametogenesis. Both groups isolated piRNAs from mouse testes, so it remains to be seen whether piRNAs are specific to spermatogenesis or whether they can also be found in oocytes. Finding piRNA targets ought to shed light on the so far elusive Piwi function.

\section{Magdalena Skipper}

ORIGINAL RESEARCH PAPERS Aravin, A. \& Gaidatzis, D. et al. A novel class of small RNAs bind to MILI protein in mouse testis. Nature 4 June 2006 (doi:10.1038/nature04916) | Girard, A. et al. A germline-specific class of small RNAs binds mammalian Piwi proteins. Nature 4 June 2006 (doi:10.1038/nature04917)

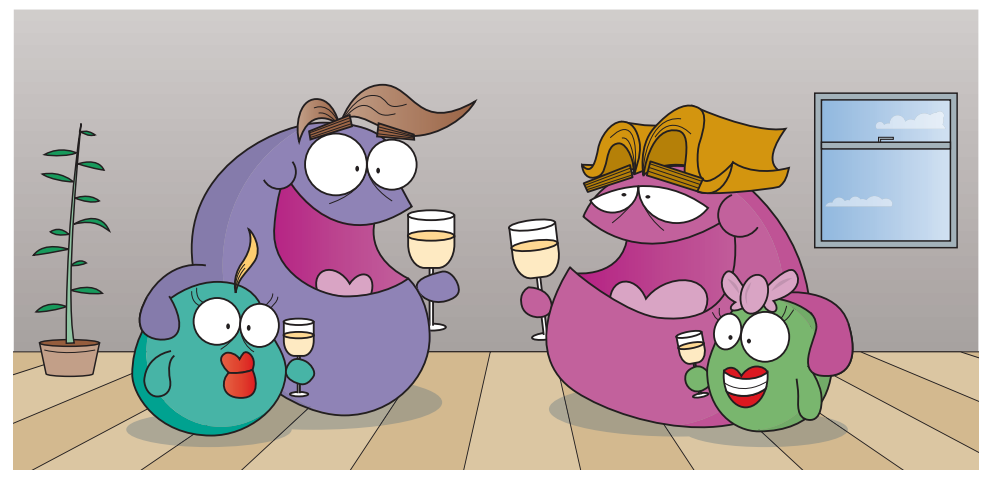

